\title{
Levantamento de tecnologias para ONGs de Proteção Animal para apoio ao resgate de animais domésticos acoplados ao ciclo de vida de um Sistema Web
}

\author{
Tatiana Tozzi \\ Instituto Federal Catarinense - \\ Campus Camboriú (IFC) \\ Rua Joaquim Garcia, s/n, Centro, \\ Camboriú, SC 88340-055 \\ tatitozzi@hotmail.com
}

\author{
Daniel Fernando Anderle \\ Instituto Federal Catarinense - \\ Campus Camboriú (IFC) \\ Rua Joaquim Garcia, s/n, Centro, \\ Camboriú, SC - 88340-055 \\ daniel.anderle@ifc.edu.br
}

\author{
Rodrigo Ramos Nogueira \\ Instituto Federal Catarinense - \\ Campus Camboriú (IFC) \\ Rua Joaquim Garcia, s/n, Centro, \\ Camboriú, SC - 88340-055 \\ rodrigo.nogueira@ifc.edu.br
}

\begin{abstract}
This paper reports on the stages of identification of existing technologies that can be used to identify and rescue lost animals and to disseminate animals for adoption by NGOs of Animal Protection, Zoonoses Center and Protective Guards. In this article the developed phases of the Course Completion Work are presented. For this, an exploratory research was carried out initially, followed by a questionnaire seeking to identify which technologies are used to identify, locate, rescue and adopt domestic animals. The current technologies found were Microchip - RFID, Microchip - NFC, Collar with qrCode, Collar with Tag, Search application, Identification application, Social networks. In this way the research reports the main technologies used to date and is going to develop an application proposal to improve and expand the dissemination of pets that are lost or for adoption.
\end{abstract}

\section{KEYWORDS}

Application model, identification of technologies, animal protection.

\section{INTRODUÇÃO}

Os animais domésticos fazem parte do dia-a-dia dos seres humanos desde os primórdios e são representados em maioria pelas espécies felina e canina. Cães e gatos são excelentes para serem companheiros dos seres humanos, além de proporcionarem conforto, amizade, e auxiliar no tratamento de doenças [4].

O Brasil possui a quarta maior população mundial de animais domésticos, segundo o IBGE de 2010, com 132,4 milhões de animais domésticos, sendo eles cães, gatos, aves, peixes e alguns animais exóticos [9]. Atualmente os animais domésticos são considerados como membros da família, conforme aponta o estudo realizado pela Associação Brasileira da Indústria de Produtos para Animais de Estimação (ABINPET) em 2016 [12].

In: XV Workshop de Trabalhos de Iniciação Científica (WTIC 2018), Salvador, Brasil. Anais do XXIV Simpósio Brasileiro de Sistemas Multimídia e Web: Workshops e Pôsteres. Porto Alegre: Sociedade Brasileira de Computação, 2018. (ㄷ) 2018 SBC - Sociedade Brasileira de Computação.

ISBN: 978-85-7669-435-9.
Segundo a Organização Mundial da Saúde (OMS), conforme pesquisa realizada em 2014, estima que o Brasil possui 30 milhões de animais abandonados, sendo 10 milhões de gatos e 20 milhões de cachorros [2].

Os animais que se encontram em situação de rua provavelmente já fizeram parte de uma família e possuíam um lar, porém vieram a ser abandonados por seus próprios donos devido a questões socioeconômicas, religiosas ou culturais [9]. Devido a isso o abandono de cães e gatos tem aumentado a população de animais em situação de rua, passando a ser um desafio para o bem-estar dos animais e à saúde pública [8]. O uso da Internet e da tecnologia atualmente é a principal fonte de divulgação de eventos de adoção de animais e para localizar animais perdidos ou abandonados.

A partir dessa constatação, este trabalho vem sendo construído com o intuito de responder a seguinte pergunta de pesquisa: "Por meio de software é possível diminuir o abandono animal e melhorar a divulgação de animais para adoção na região da AMFRI ${ }^{1}$ ". Para atingir o objetivo este trabalho visa identificar as tecnologias utilizadas atualmente para auxiliar no resgate, identificação e divulgação de animais domésticos, tendo como base o seguinte roteiro:

- Apresentar os resultados através de uma Pesquisa de opinião;

- Descrever as tecnologias encontradas, classificá-las e identificar a aplicabilidade de uso;

- Desenvolver um projeto para o desenvolvimento de um Sistema Web para apoio às ONGs e Protetores Independentes;

- Testar a viabilidade do modelo proposto junto às ONGs e Protetores Independentes.

\section{METODOLOGIA}

Este trabalho se classifica quanto à natureza aplicada e tecnológica, em questão aos objetivos como exploratória e quanto aos procedimentos bibliográfica, conforme identificado

\footnotetext{
${ }^{1}$ Associação dos Municípios da Foz do Rio Itajaí - Santa Catarina, composta por 11 municípios (Balneário Camboriú, Balneário Piçarras, Bombinhas, Camboriú, Ilhota, Itajaí, Itapema, Luiz Alves, Navegantes, Penha e Porto Belo) [1].
} 
por meio da Figura 1 - imagem A.

A metodologia adotada para o desenvolvimento deste trabalho foi dividida em seis fases, as quais estão relacionadas com os objetivos propostos desta pesquisa. A Figura 1 - imagem B, mostra as etapas que foram empregadas na metodologia de desenvolvimento deste trabalho.

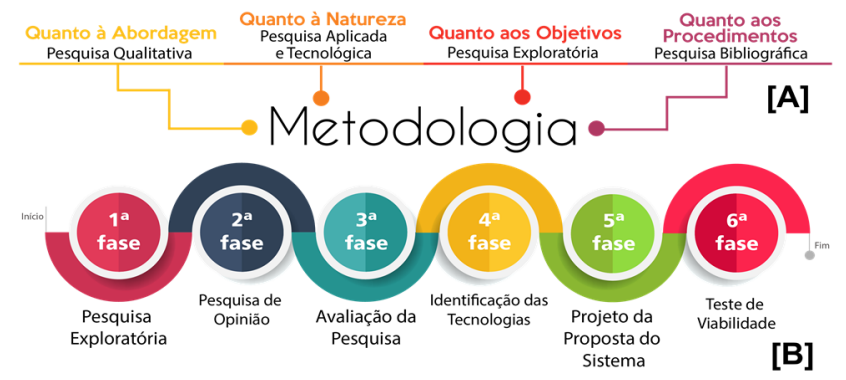

Figura 1: Metodologia [A] e Fases de desenvolvimento [B].

A primeira fase correspondeu na Pesquisa Exploratória, em bases de dados, livros, páginas Web e trabalhos relacionados buscando identificar tecnologias que possam auxiliar a localização de animais perdidos ou abandonados. Na segunda fase foi desenvolvido e aplicado a Pesquisa de Opinião na região da AMFRI. Na terceira foram avaliados os resultados da Pesquisa de Opinião. Na quarta fase consiste da identificação das tecnologias encontradas na Pesquisa de Opinião e nos Trabalhos relacionados.

A quinta fase será desenvolvido um projeto de Sistema Web, na qual serão estabelecidos os requisitos do sistema, os atores; desenvolvido os casos de uso, a modelagem do banco de dados e a prototipação das telas do sistema. A sexta fase constituirá de testar a viabilidade da aplicação proposta junto às ONGs, Centros de Zoonoses e Protetores Independentes, identificando se nossa proposta vem atender necessidades das mesmas para a divulgação, identificação, localização e adoção de animais domésticos.

\section{FASES DE DESENVOLVIMENTO}

Até o presente momento foram desenvolvidas quatro fases do trabalho, as quais serão apresentadas resumidamente a seguir.

\subsection{Pesquisa exploratória}

Esta fase teve como pretensão identificar os principais conceitos abordados durante o decorrer da pesquisa, os quais são: animais domésticos, posse responsável, Protetores Independentes, ONG, Centro de Zoonoses, resgate de animais, abandono de animais. Como parte desta fase foram selecionados seis trabalhos científicos (artigos e monografias). Por meio destes trabalhos buscou-se identificar outros projetos que foram desenvolvidos tendo como objetivo auxiliar os animais domésticos, desde um sistema informatizado para uma ONG, como a criação de um registro nacional de identificação de animais. Estes trabalhos e as tecnologias e a tecnologia proposta são apresentadas na subseção 3.4 - Identificação das tecnologias.

\subsection{Pesquisa de opinião}

Está fase consistiu em desenvolver uma pesquisa de opinião com os moradores da região da AMFRI, por meio de um questionário composto por 24 perguntas utilizando o Google Forms. Antes da aplicação da pesquisa no público-alvo, uma busca foi realizada no Google e Facebook para localizar as ONGs e Protetores Independentes da região da AMFRI. Foram identificados 51 páginas e grupos no Facebook e 3 sites. Para cada um dos grupos, páginas e sites uma mensagem foi enviada com um convite para participar da pesquisa.

As perguntas tinham como objetivo identificar à faixa etária, o sexo, a quantidade de animais e a espécie de animais possuem, se os participantes da pesquisa fazem parte de alguma ONG de Proteção Animal ou se atua como Protetor Independente, quais tecnologias já usaram para divulgar animais abandonados ou para adoção entre outras questões.

\subsection{Avaliação da pesquisa}

Esta fase apresenta os principais resultados da pesquisa. Após duas semanas de divulgação e aplicação da pesquisa de opinião, 100 pessoas responderam à pesquisa. As cidades que mais participaram da pesquisa foram as cidades, Camboriú com $45 \%$ e Balneário Camboriú com $32 \%$. A maioria dos participantes tem entre 26 a 31 anos (24\%), sendo que $69 \%$ das participações foram de mulheres. $89 \%$ afirmaram ter animais domésticos.

Dos participantes, $71 \%$ já utilizaram alguma tecnologia para auxiliar no resgate e divulgação de animais domésticos. A pergunta em seguida continha várias tecnologias ${ }^{2}$, a qual os participantes poderiam selecionar quais já usaram, sendo a mais identificada as Redes Sociais. Questionados se gostariam de conhecer as tecnologias citadas na pesquisa, 93\% responderam que sim, uma vez que $61 \%$ dos participantes não conheciam as tecnologias citadas. Por meio da $19^{\mathrm{a}}$ pergunta, descobrimos que $77 \%$ dos participantes utilizam redes sociais para auxiliar na divulgação de animais perdidos, abandonados ou para adoção. Sendo o Facebook, a rede social mais utilizada para este fim e as publicações desses anúncios são em maioria feitas na própria linha do tempo dos participantes. $98 \%$ dos participantes acham que um Sistema Web poderia melhorar e ampliar a divulgação de animais perdidos ou para adoção.

Como forma de devolutiva para os participantes da pesquisa foram elaborados dois infográficos, o primeiro contendo as respostas utilizando gráficos e o segundo (figura 2) apresenta os principais dados das respostas. Ambos infográficos foram enviados por e-mail aos participantes e publicado no Facebook como forma de agradecimento.

\subsection{Identificação das Tecnologias}

Nesta fase foram abordadas as tecnologias identificadas na Pesquisa de opinião e nos Trabalhos relacionados.

\subsubsection{Tecnologias identificadas na Pesquisa de Opinião.}

\footnotetext{
${ }^{2}$ Microchip - RFID, Microchip - NFC, Coleira com qrCode, Coleira com Tag, Aplicativo de busca, Aplicativo de Identificação, Redes sociais.
} 
Levantamento de tecnologias para ONGs de Proteção Animal para apoio ao resgate de animais domésticos acoplados ao ciclo de vida de um Sistema Web

O microchip RFID ou Radio-Frequency IDentification (Identificação por rádio frequência) é um método de identificação automática por meio de sinais de rádio. Os sinais são recuperados e armazenados dados remotamente por meio de um dispositivo de tags ou etiqueta RFID. A tag ou etiqueta é um transponder ou microchip que pode ser colocado em uma pessoa, animal, produto ou embalagem. Microchip NFC (Near Fiel Communication) é uma tecnologia que possibilita a troca de informações e dados entre dispositivos. Para que ocorra a troca de informações é apenas necessário que ambos dispositivos estejam até 10 centímetros próximos um do outro, tornando essa transmissão de dados segura [5].

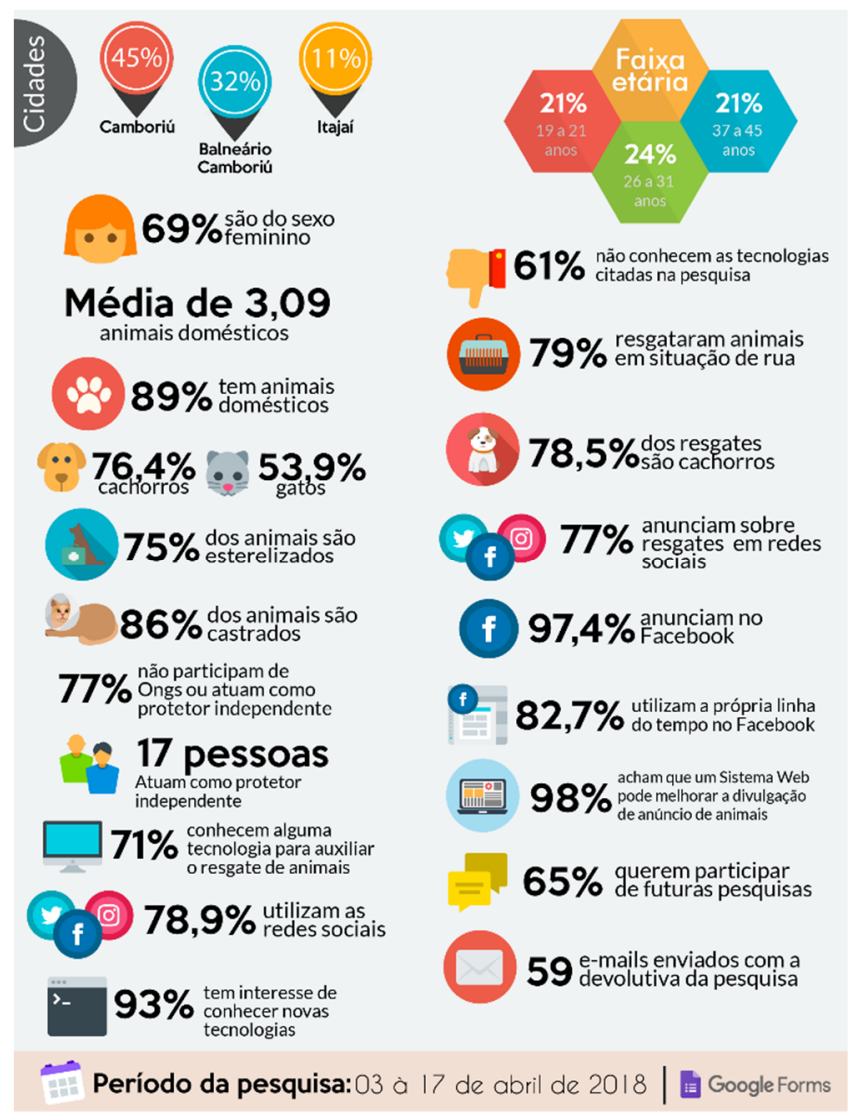

Figura 2: Infográfico com os dados principais da pesquisa.

A coleira com qrCode (Quick Response Code), consiste de uma coleira com uma medalha de identificação com qrCode. A medalha de alumínio possui um qrCode que possibilita acessar a página do animal. Por meio da página a pessoa que venha encontrar o animal com esta coleira pode ter acesso as informações de contato do tutor, telefone do médico veterinário, fotos e informações médicas. Além do qrCode a medalha pode conter o endereço (link) da página do animal, caso quem tenha encontrado o animal não tenha acesso a um dispositivo que lê o qrCode. A coleira com $\boldsymbol{t a g}$ é uma alternativa para utilização do microchip sem que este seja implantado no animal. A tag contendo os dados do animal (o código de identificação) é
WebMedia 2018: Workshops e Pôsteres, Salvador, Brasil

colocado na coleira do animal, e caso ele venha se perder pode-se utilizar um leitor para obter essa informação e promover o reencontro entre animal e tutor.

Aplicativos de busca podem ser utilizados para cadastrar animais que tenham fugido de casa ou se perderam de seus tutores. Através deste cadastro os tutores inserem fotos e informações do animal desaparecido e dados de contato no aplicativo. Já Aplicativo de identificação promovem a identificação do animal por meio de reconhecimento facial, utilizando a tecnologia de comparação de imagens (visão computacional e inteligência artificial). Como citado anteriormente a rede social mais utilizadas pelos brasileiros é o Facebook e além desta rede social as demais redes também são grandes aliadas na procura de animais perdidos, porém em fevereiro de 2018 foi criada da rede social Puppyfi, com o principal objetivo auxiliar os animais, assim auxiliando tutores a encontrarem seus animais desaparecidos e encaminhar os que foram abandonados para adoção.

3.4.2 Tecnologias identificada nos trabalhos relacionados.

No artigo de Carpanezi, Tomazela e Pontes [3] apresentam o desenvolvimento de um aplicativo, onde foram cadastrados os animais para adoção. A aplicação possibilita quem tivesse interesse em adotar algum dos animais cadastrados possa conhecer as características do mesmo de forma fácil e rápida.

Já Silva, Franco e Molina [13] teve como proposta à implementação de um sistema informatizado para registro de controle dos animais acolhidos pelo Centro de Controle de Zoonoses. O Projeto Adoção Animal de Evangelista et al. [7] teve como pretensão ampliar a divulgação dos animais abandonados que vivem em abrigos utilizando mídias sociais [Facebook e Blogger] e outros recursos físicos, buscando incentivar a adoção de animais.

No trabalho de Menezes Filho e Souza [11] teve como objetivo o desenvolver uma ferramenta para registro e identificação de animais de companhia. Foram utilizadas as tecnologias para o desenvolvimento web (FHipster, Spring, Angular e Bootstrap), como a intenção de criar uma base de dados para as prefeituras armazenarem informações sobre a população de animais e promover ações estratégicas para controle da populacional.

$\mathrm{Na}$ monografia de Donatti [6] foram desenvolvidos dois sistemas utilizando um protocolo baseado no ZigBee e a tecnologia Global Positioning System - GPS. Ambos sistemas foram criados para coletar dados referentes ao posicionamento global dos animais (bovinos), identificando a longitude e latitude do animal.

3.4.3 Classificação das tecnologias. Conforme a tabela 1, a coluna 1 contém as tecnologias encontradas, na coluna 2 e 3

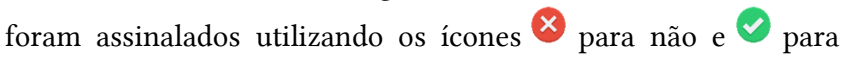
sim, as tecnologias classificadas como Hardware e Software respectivamente. Algumas das tecnologias listadas na tabela 2, são consideradas hardware e software pois uma demanda da outra para total funcionalidade da tecnologia. 
Tabela 1. Classificação das tecnologias

\begin{tabular}{|c|c|c|}
\hline Tecnologias & Hardware & Software \\
\hline Microchip RFID & 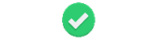 & ( \\
\hline Microchip NFC & 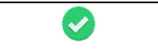 & 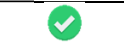 \\
\hline Coleira com qrCode & $x$ & 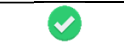 \\
\hline Coleira com Tag & 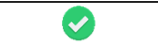 & 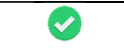 \\
\hline Aplicativo de Busca & $x$ & ( \\
\hline Aplicativo de Identificação & $x$ & 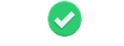 \\
\hline Redes sociais & $x$ & 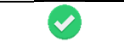 \\
\hline Aplicativo mobile para adoção & $\otimes$ & 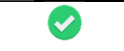 \\
\hline $\begin{array}{l}\text { Informatização de Centro de } \\
\text { Zoonoses }\end{array}$ & $x$ & ( \\
\hline $\begin{array}{l}\text { Facebook e Blogger como ferramenta } \\
\text { de divulgação }\end{array}$ & 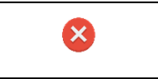 & 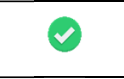 \\
\hline Registro geral de animais & $\otimes$ & ( \\
\hline $\begin{array}{l}\text { Monitoramento de animais com } \\
\text { rede de sensores sem fio }\end{array}$ & 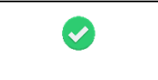 & ( \\
\hline
\end{tabular}

3.4.4 Aplicabilidade das tecnologias

Segundo a tabela 2, a coluna 1 representa as tecnologias encontradas, na coluna 2 as ONGs, na coluna 3 os Centros de Zoonoses e na coluna 4 os Protetores Independentes. Foram assinados utilizando os ícones $\times$ para não e $\bullet$ para sim.

Tabela 2. Aplicação das tecnologias

\begin{tabular}{|c|c|c|c|}
\hline Tecnologias & ONGs & $\begin{array}{c}\text { Centros de } \\
\text { Zoonoses }\end{array}$ & $\begin{array}{c}\text { Protetores } \\
\text { Independentes }\end{array}$ \\
\hline Microchip RFID & 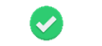 & $\theta$ & 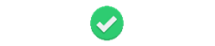 \\
\hline Microchip NFC & 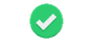 & 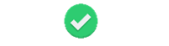 & $\nabla$ \\
\hline $\begin{array}{l}\text { Coleira com } \\
\text { qrCode }\end{array}$ & $x$ & $x$ & $\varnothing$ \\
\hline Coleira com Tag & $x$ & $x$ & $\theta$ \\
\hline $\begin{array}{l}\text { Aplicativo de } \\
\text { Busca }\end{array}$ & $\theta$ & $\theta$ & 0 \\
\hline $\begin{array}{l}\text { Aplicativo de } \\
\text { Identificação }\end{array}$ & 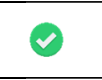 & $\theta$ & 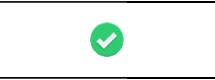 \\
\hline Redes sociais & 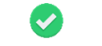 & 0 & 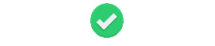 \\
\hline $\begin{array}{l}\text { Aplicativo mobile } \\
\text { para adoção }\end{array}$ & 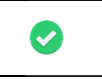 & 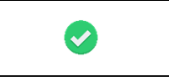 & 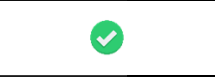 \\
\hline $\begin{array}{l}\text { Informatização de } \\
\text { Centro de } \\
\text { Zoonoses }\end{array}$ & 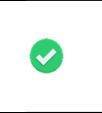 & 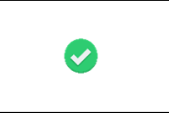 & $x$ \\
\hline $\begin{array}{l}\text { Facebook e Blogger } \\
\text { como ferramenta } \\
\text { de divulgação }\end{array}$ & 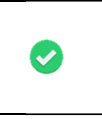 & 2 & 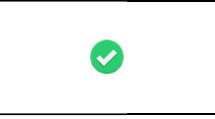 \\
\hline $\begin{array}{l}\text { Registro geral de } \\
\text { animais }\end{array}$ & 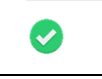 & 0 & $\nabla$ \\
\hline $\begin{array}{l}\text { Monitoramento de } \\
\text { animais com rede } \\
\text { de sensores sem fio }\end{array}$ & 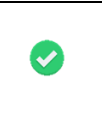 & 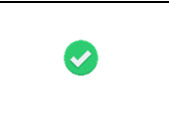 & $x$ \\
\hline
\end{tabular}

\section{CONSIDERAÇÕES FINAIS E TRABALHOS FUTUROS}

Espera-se que este trabalho possa contribuir para a conscientização da importância da adoção responsável de animais domésticos e no desenvolvimento de uma aplicação que possa auxiliar o resgate, localização, identificação e adoção de animais domésticos.

Este artigo visa apresentar as etapas que foram realizadas e as que serão desenvolvidas no decorrer do Trabalho de Conclusão de Curso. Assim, o feedback da comunidade do Workshop de Trabalhos de Iniciação Científica é de grande importância para refinar e aprimorar esta pesquisa, assim levando a uma conclusão satisfatória. Já como trabalhos futuros pretende-se dar seguimento as duas fases finais $\left(\begin{array}{lll}5 & \text { e } & 6\end{array}\right)$ e iniciar o desenvolvimento da aplicação.

\section{REFERENCIAS}

[1] AMFRI. Associação dos Municípios da Foz do Rio Itajaí. Acessado em 7 Mai. 2018 de https://www.amfri.org.br/

[2] ANDA. Brasil tem 30 milhões de animais abandonados. 2014. Acessado em 14 Set. 2017 de https://anda.jusbrasil.com.br/noticias/100681698/brasil-tem30-milhoes-de-animais-abandonados

[3] CARPANEZI, C. A., et al. Desenvolvimento de um aplicativo mobile para adoção de animais de estimação. 2016. Acessado em 28 fev. 2018 de http://reverte.fatecid.com.br/index.php/revista/article/view/183

[4] CARVALHO, L. 9 benefícios que bichos de estimação trazem à saúde. 2016 Acessado em 13 Set. 2017 de https://exame.abril.com.br/estilo-de-vida/9beneficios-que-bichos-de-estimacao-trazem-a-saude/

[5] COIMBRA, D. da S. O Uso da Tecnologia NFC na Identificação PET. 2016. Acessado em 28 fev. 2018 de http://www2.uesb.br/computacao/wpcontent/uploads/2014/09/O-Uso-da-Tecnologia-NFC-daIdentifica\%C3\%A7\%C3\%A3o-PET-DIEGO-COIMBRA.pdf

[6] DONATTI. R. N. Desenvolvimento de um sistema de monitoramento de animais, utilizando rede de sensores sem fio, baseado no protocolo ZigBee e tecnologia GPS. 2017. Acessado em 10 mai. 2018 de http://www.teses.usp.br/teses/disponiveis/74/74134/tde-14062017112910/publico/ME8845716COR.pdf

[7] EVANGELISTA, A. G., et al. Projeto adoção animal: Incentivando a prática da adoção de Cães e Gatos Abandonados - Resultados preliminares. 2015. Acessado em 10 mai. 2018 de http://eventos.ifc.edu.br/wpcontent/uploads/sites/5/2015/10/PROJ

ETO-ADO $\%$ C3\%87\%C3\%83O-ANIMAL-IFC-INCENTIVANDO-APR\%C3\%81TICA-DA-ADO\%C3\%87\%C3\%83O-DE-C\%C3\%83ES-E-GATOSABANDONADOS-RESULTADOS-PRELIMINARES.pdf

[8] GARCIA, R. C. M. Normas e políticas públicas para controle populacional de cães e gatos. In: CONGRESSO BRASILEIRO DE BIOÉTICA E BEM-ESTAR ANIMAL, 3., 2014, Curitiba: Anais...Curitiba: UFPR/LABEA, 2014. p. 149.

[9] IBGE. Um panorama da saúde no Brasil: acesso e utilização dos serviços, condições de saúde e fatores de risco e proteção à saúde. 2010. Acessado em 13 nov. 2017 de http://bvsms.saude.gov.br/bvs/publicacoes/pnad_panorama_saude_brasil.pd $\mathrm{f}$

[10] MELO, L. Como o brasileiro cuida e quanto gasta com seus pets. 2016. Acessado em 21 Out. 2017 de https://exame.abril.com.br/negocios/como-obrasileiro-cuida-e-quanto-gasta-com-seus-pets/

[11] MENEZES FILHO, C. T. B et al. Registro geral de Animais (RGA): um sistema para o registro e identificação de animais de companhia. 2017. $\begin{array}{llllll}\text { Acessado } & \mathrm{em} & 12 & \mathrm{abr} & 2018 & \mathrm{de}\end{array}$ https://repositorio.ufsc.br/xmlui/bitstream/handle/123456789/176967/RGA.p df

[12] REZENDE, L. F. G., et. al. Perfil dos proprietários de cães e gatos e a prática da guarda responsável dos acadêmicos CEULJI-ULBRA. Archives of Veterinary Science, v. 17, p.34-36, resumo 012, 2012

[13] SILVA, et al. Sistema Informatizado para o Centro de Controle de Zoonoses - Americana/SP. 2017. Acessado em 10 mar. 2018 de https://febrace.org.br/mostra3m2017/_data/pdf/3M_anaisVMostra.pdf/ 